\title{
La inteligencia emocional como una competencia básica en la formación inicial de los docentes: algunas evidencias
}

\section{Raquel Palomera ${ }^{1}$, Pablo Fernández-Berrocal ${ }^{2}$, Marc A. Brackett ${ }^{3}$}

${ }^{1}$ Departamento de Educación, Universidad de Cantabria, Santander ${ }^{2}$ Departamento de Psicología Básica, Universidad de Málaga, Málaga ${ }^{3}$ Personality and Social Psychology, Yale University, New Haven, CT

España/E.E.U.U. 


\section{Resumen}

Este artículo reivindica la inclusión de las competencias emocionales como competencias básicas en la escolaridad obligatoria y en los objetivos de la formación inicial del profesorado que se está diseñando actualmente dentro del marco del Espacio Europeo de Educación Superior (EEES). Para ello, se realiza una revision de las investigaciones más actuales en torno al papel fundamental que tienen las competencias emocionales y, más concretamente, la inteligencia emocional (IE; Mayer y Salovey, 1997) sobre el funcionamiento personal, social y académico de los alumnos, así como sobre la efectividad y bienestar del docente. Además, se analizan las multiples funciones que la legislación educativa vigente demanda al maestro. De esta manera, por medio de la exposición de evidencias científicas sobre la validez predictiva de la inteligencia emocional y su relación con los objetivos educativos actuales, este trabajo pretende demostrar la necesidad de desarrollar las competencias emocionales en los docentes con el fin de promover su bienestar y rendimiento laboral, así como el de sus futuros alumnos. Para ello, proponemos la formación inicial del profesorado como medio prioritario para dicho aprendizaje, así como requisito para la posterior e inevitable formación permanente.

Palabras Clave: inteligencia emocional, competencias básicas, formación inicial, docentes, escuela. 


\begin{abstract}
This article calls for the inclusion of emotional competencies within basic competencies considered in compulsory schooling and in the objectives of pre-service teacher training that is now being designed within the European Space for Higher Education. Toward this end, we review current research on the fundamental role of emotional competencies, concretely, emotional intelligence (EI; Mayer and Salovey, 1997), on students' personal, social and academic functioning, as well as on teacher effectiveness and well-being. In addition, we analyze the multiple functions that educational legislation requires of the teacher. By presenting the scientific evidence for the predictive validity of emotional intelligence and its relation to present educational objectives, this paper seeks to demonstrate the need for developing emotional competencies in teacher education in order to enhance well-being and job performance, both in teachers as well in their future students. We propose pre-service teaching training as the priority educational context for this type of learning, which is also an indispensable requirement for later ongoing professional development.
\end{abstract}

Keywords: emotional intelligence, basic competencies, initial education, teachers, school

Received: 05/16/08 Initial Acceptance: 06/19/08 Final Acceptance: 07/02/08 


\section{Introducción}

La pretensión de los países que integran la Comunidad Europea de convertirse en una sociedad basada en el conocimiento, más competitiva y dinámica, y con una mayor cohesión social, se ha plasmado en la formulación de unos objetivos educativos comunes. Por ello, estamos asistiendo a profundos cambios en el sistema educativo español en todos sus niveles de formación, que se han traducido en el desarrollo de nuevas leyes educativas. Asistimos así al proceso de transformación de los títulos universitarios y planes de estudio con el objeto de conseguir la cercana integración en el Espacio Europeo de Educación Superior (EEES), y al paralelo proceso de modificación de la escolaridad obligatoria (LOE, 2006), ambas orientadas al desarrollo integral centrado en competencias, a la calidad educativa y a dar respuesta a las demandas socio-profesionales actuales. Estamos en un proceso de cambio y por ello de oportunidades para mejorar la oferta formativa y sus resultados.

Desde finales del siglo XX ha surgido un gran interés por el papel que juega la afectividad y las emociones en la educación. Los diferentes profesionales que integran el sistema educativo han comprendido la importancia de los sentimientos en el desarrollo integral de sus alumnos y en su propio quehacer diario por lo que reclaman la necesidad de promover no sólo el desarrollo de los niños y jóvenes a nivel académico sino también desarrollar las competencias sociales y emocionales de éstos (Elias et al., 1997; Greenberg et al., 2003).

Aunque los vínculos existentes entre estas áreas de desarrollo no estaban bien consolidados, un reciente estudio de meta-análisis de cerca de 300 investigaciones ha mostrado que la educación socio-emocional no sólo aumenta el aprendizaje en estas áreas del desarrollo sino también el aprendizaje académico (Durlak y Weissberg, 2005). Por otro lado, la competencia emocional de los docentes es necesaria para su propio bienestar personal y para su efectividad y calidad a la hora de llevar a cabo los procesos de enseñanza-aprendizaje del aula, en general, y del desarrollo socio-emocional en los alumnos, en particular (Sutton y Wheatly, 2003).

Siguiendo el trabajo impulsado por el grupo The Collaborative for Academic, Social, and Emotional Learning (CASEL) en Estados Unidos, el cual lleva promocionando la inclusión socio-emocional en las escuelas desde hace más de dos décadas, el Departamento de 
Educación y Habilidades del Gobierno de Gran Bretaña ha llevado a cabo un estudio buscando el mismo fin. El trabajo realizado consistía en identificar las metodologías más efectivas para desarrollar habilidades socio-emocionales en los niños y en implementar un programa de promoción de la inclusión, cohesión social, aprendizaje y bienestar emocional infantil mediante la educación de estas competencias socio-emocionales en los niños/as llamado 'Every Child Matters' (DfES, 2004). De igual manera, se ha atendido a la formación del profesorado para el desarrollo de dichas competencias, partiendo de la idea de que no es posible enseñar una competencia que previamente no se ha alcanzado, al igual que no es posible enseñar con calidad ante la ausencia de bienestar docente. El estudio concluye con la recomendación de desarrollar explícitamente las competencias tanto sociales como emocionales no sólo en la escuela sino también en las instituciones dirigidas a la formación del profesorado (Weare y Grey, 2003).

Siguiendo las conclusiones del modelo educativo inglés, este trabajo se propone reivindicar el lugar que deberían ocupar las competencias emocionales en la educación básica y, por tanto, en la formación inicial del profesorado, mediante una breve revisión de las investigaciones más recientes en torno a las aportaciones de la inteligencia emocional para explicar el funcionamiento humano y su adaptación exitosa, y mediante un análisis del perfil profesional demandado hoy en día al docente.

\section{Relevancia actual de las competencias emocionales}

La percepción, utilización, comprensión, y regulación de las emociones, así como sus implicaciones en nuestra vida diaria han sido estudiadas desde hace décadas, aunque han suscitado un interés creciente en los últimos años (Bar-On y Parker, 2000; Ciarrochi, Forgas y Mayer, 2001; 2006; Fernández-Berrocal y Ramos, 1992). Después de asumir durante siglos la dicotomía cartesiana imperante en Occidente en la que la razón aparece separada de la emoción, la ciencia ha demostrado la falacia de tal dicotomía (Damasio, 1994; Forgas, 2000). Diversos estudios han explicado cómo la emoción afecta no sólo a los contenidos del pensamiento sino también a los propios procesos implicados en éste (Bless, 2000) y en las interacciones sociales (Lupton, 1998).

Las implicaciones de estos resultados en la tarea diaria de los docentes son fáciles de deducir. Los profesores son conscientes del papel que juegan las emociones en su labor diaria. 
Las emociones y las habilidades relacionadas con su manejo, afectan a los procesos de aprendizaje, a la salud mental y física, a la calidad de las relaciones sociales y al rendimiento académico y laboral (Brackett y Caruso, 2007). La docencia es considerada una de las profesiones más estresantes, sobre todo porque implica un trabajo diario basado en interacciones sociales en las que el docente debe hacer un gran esfuerzo para regular no sólo sus propias emociones sino también las de los estudiantes, padres, compañeros, etc. (Brotheridge y Grandey, 2002). Los profesores, desafortunadamente, experimentan con más frecuencia emociones negativas que positivas (Emmer, 1994). Las emociones negativas, como por ejemplo la ansiedad, interfieren en nuestra capacidad cognitiva para el procesamiento de la información (Eysenck y Calvo, 1992) mientras que las positivas, aumentan nuestra capacidad creativa para generar nuevas ideas y por tanto nuestra capacidad de afrontamiento ante las dificultades (Frederickson, 2001). Las emociones positivas de los profesores pueden aumentar el bienestar docente y también el ajuste de sus alumnos (Birch y Ladd, 1996). Este afecto positivo además puede formar una espiral que a su vez facilite un clima de clase más favorecedor para el aprendizaje (Sutton y Whealey, 2003). Es por ello que la capacidad de identificar, comprender y regular las emociones, tanto positivas como negativas, se hace imprescindible en esta profesión, para poder utilizar y generar las emociones a nuestro favor.

Gran parte de la expectación sobre las aportaciones que el estudio de las emociones puede ofrecer para la mejora de la educación se debe a la irrupción de un nuevo ámbito de estudio denominado Inteligencia Emocional (IE). Desde que Salovey y Mayer acuñaron el término en 1990, el campo de estudio de la IE ha generado un progresivo desarrollo de trabajos e investigaciones. En la actualidad, el debate sobre la propia definición teórica del constructo, su validez discriminante respecto a otros constructos clásicos de la psicología o el desarrollo de instrumentos de evaluación fiables sustenta gran parte de los trabajos que se llevan a cabo. Por otro lado, una gran línea de investigación se dirige a conocer los efectos de la IE en diferentes contextos aplicados. Una de las consecuencias del desarrollo de este ámbito de investigación ha sido la mayor concienciación social sobre la importancia del uso adecuado de las emociones. Esto se ha traducido en un aumento en la demanda de formación en competencias emocionales tanto en el contexto educativo como en el resto de ámbitos profesionales.

La formación en competencias emocionales es necesaria para que tanto los niños (futuros adultos) como el profesorado puedan adaptarse con éxito. Pero no es sólo importante de 
cara a poder desarrollar dichas competencias en los estudiantes o a prevenir desajustes en la salud mental del profesorado, sino también para crear entornos favorecedores de aprendizaje.

Los estudios llevados a cabo en la línea de la psicología positiva reclaman la necesidad de generar climas de seguridad y emociones positivas en el aula para facilitar el desarrollo y bienestar o felicidad de los alumnos (Seligman, 2005). Según la clásica definición de Diener, Sandvik y Pavot (1991), la felicidad se refiere a la experiencia de emociones positivas en un gran porcentaje de tiempo, respecto a la cantidad de tiempo en que sentimos emociones negativas y, hoy en día, sabemos que las emociones positivas favorecen el aprendizaje y la consecución de relaciones interpersonales significativas (Lyubomirsky, Diener y King, 2005), entre otros muchos beneficios. Un clima escolar adecuado se ha observado que presenta efectos positivos sobre el ajuste psicológico de los estudiantes (Kuperminc, Leadbeater y Blatt, 2001; Roeser y Eccles, 1998), asociándose a un desarrollo saludable, un aprendizaje óptimo y disminuyendo las conductas desadaptativas (Kuperminc, Leadbeater, Emmons y Blatt, 1997; Westling, 2002). Por tanto, parece importante asegurarnos de que los docentes sean capaces de crear un ambiente positivo en sus aulas para fomentar el desarrollo y aprendizaje, además del bienestar de los alumnos.

\section{Aportaciones de la Inteligencia emocional desde el modelo de habilidad}

La perspectiva teórica de la que partimos en este trabajo está basada en el modelo de IE de Mayer y Salovey (1997) que, por otra parte, constituye el planteamiento científico con más apoyo empírico y con una base teórica más fundamentada (Mayer, Caruso y Salovey, 1999; Mayer, Salovey y Caruso, 2000). Desde este modelo, la IE engloba un conjunto de habilidades relacionadas con el procesamiento emocional de la información. En concreto, la definición más extendida describe la IE como la habilidad para percibir, valorar y expresar emociones con exactitud; la habilidad para acceder y/o generar sentimientos que faciliten el pensamiento; la habilidad para comprender emociones y el conocimiento emocional y la habilidad para regular las emociones promoviendo un crecimiento emocional e intelectual (Mayer y Salovey, 1997).

Existen evidencias de que la IE es un predictor significativo del funcionamiento social y personal del individuo (Extremera y Fernández-Berrocal, 2005a; Schutte et al., 2001). Una de las líneas de investigación más activas trata de establecer la utilidad predictiva de la IE 
sobre diversas áreas vitales de los jóvenes (para una revisión más amplia ver FernándezBerrocal, Extremera, y Palomera, 2008). Por ejemplo, la IE se ha encontrado relacionada con un menor índice de comportamientos desajustados, como conductas disruptivas y agresivas (Bohnert, Cmic y Lim, 2003; Gil-Olarte, Palomera y Brackett, 2006), conductas de riesgo, como el consumo de drogas (Trinidad y Johnson, 2002) o la conducción temeraria (Brackett, Mayer y Warner, 2004). Pero sobre todo, se ha encontrado relacionada de forma significativa y positiva con un mayor comportamiento adaptado como: mayor calidad en las relaciones sociales (Gil-Olarte, Palomera y Brackett, 2006; Lopes, Brackett, Nezlek, Schütz, Sellin y Salovey, 2004; Lopes, Salovey y Straus, 2003), mayor retención en el sistema educativo (Parker, Hogan, Eastabrook, Oke y Word, 2006), comportamiento prosocial (Lopes, Salovey, Côté y Beers, 2005), un mejor rendimiento académico (Brackett, Mayer y Warner, 2004; GilOlarte, Palomera y Brackett, 2006), más satisfacción con la vida (Extremera y FernándezBerrocal, 2005b; Palmer, Donaldson y Stough, 2002; Palomera y Brackett, 2006), utilización de estrategias de afrontamiento más adaptativas (Fernández-Berrocal y Extremera, 2006; Gohm y Clore, 2002), mejor salud mental (Fernández-Berrocal, Alcaide, Extremera y Pizarro, 2006) y una mayor capacidad para interrumpir estados emocionales negativos y prolongar los positivos (Salovey, Stroud, Woolery y Epel, 2002; Williams, Fernández-Berrocal, Extremera, Ramos y Joiner, 2004). Los niños con alta IE son evaluados por sus pares y profesores de forma más positiva, como niños con un comportamiento más prosocial y menos agresivo, dependiente o intimidatorio con los otros (Petrides, Sangareau, Furnham y Frederickson, 2006). También, los estudiantes con mayor IE afrontan mejor la transición de la escuela al centro de educación secundaria, con mejores resultados académicos, autovaloración, mayor asistencia y ajuste comportamental en comparación con sus compañeros con baja IE (Qualter, Whiteley, Hutchinson y Pope, 2007).

Recientemente se ha comenzado a analizar la relación entre la IE y el ajuste personal y bienestar del docente. En un trabajo llevado a cabo con profesores de secundaria en Inglaterra, se ha observado que la IE del profesor predice el nivel de burnout que sufren (Brackett, Palomera y Mojsa, en preparación), confirmando un reciente estudio en el que aparecía relacionada la habilidad de los docentes para regular las emociones con los niveles de despersonalización, autorrealización y desgaste emocional que percibían (Mendes, 2003). Al mismo tiempo los docentes con alta IE utilizan estrategias de afrontamiento más positivas y adaptativas ante diversas fuentes de estrés escolar y perciben una mayor satisfacción laboral. Dicha influencia de la IE sobre los niveles de estrés y satisfacción laboral aparece mediada por el 
mayor grado de afecto positivo que experimenta el profesor y el apoyo de los directores de centro (Brackett, Palomera, y Mojsa, en preparación).

El burnout ha mostrado tener repercusiones negativas no sólo sobre el bienestar del docente sino también sobre los procesos de enseñanza-aprendizaje en los que está inmerso. Estudios previos muestran cómo el burnout influye negativamente sobre el rendimiento de los alumnos y la calidad de su enseñanza (Vanderberghe y Huberman, 1999) y afecta negativamente a las relaciones interpersonales profesor-alumno (Yoon, 2002). No es de extrañar, por tanto, que el profesorado identifique la habilidad de regular sus emociones como una competencia imprescindible para poder conseguir las metas académicas, construir relaciones sociales positivas y controlar los procesos del aula (Sutton y Harper, en prensa). Por otra parte, el entrenamiento en competencias emocionales de los profesores noveles ha mostrado su efectividad no sólo en el aumento de su propia competencia emocional, sino también en la predicción de una transición ajustada del rol de estudiante a la vida profesional (Byron, 2001).

\section{Perfil profesional del docente actual}

Conocer el perfil profesional del docente actual, desde un modelo educativo descriptivo, implica analizar las competencias que debería desarrollar para responder a las demandas de la sociedad en nuestro contexto específico y en este momento histórico en concreto.

Para el diseño de la formación inicial de los maestros, así como en la formación de postgrado dirigida a la formación de profesores de secundaria, es necesario conocer los objetivos respecto a qué contenidos y competencias básicas han de desarrollar los futuros docentes en cada uno de sus alumnos. El estudio de las competencias básicas no es nuevo, aunque su historia es relativamente reciente y se ha desarrollado a un ritmo rápido en los últimos años: desde la Conferencia Mundial de Jomtien (1990) en la que se formuló la Declaración Mundial sobre "Satisfacción de las Necesidades Básicas de Aprendizaje", hasta el desarrollo del Proyecto DeSeCo (Rychen y Salganick, 2001) y PISA (OCDE, 2002), o el Eurydice (2002) por citar los proyectos más relevantes en nuestro contexto europeo, con el fin de evaluar y promocionar la efectividad y calidad de la educación. 
Durante todo este proceso, se han utilizado diversos términos para referirse al conjunto de habilidades cognitivas, procedimentales y actitudinales que pueden y deben ser alcanzadas a lo largo de la educación obligatoria por la mayoría del alumnado y que resultan imprescindibles para garantizar el desenvolvimiento personal y social y la adecuación a las necesidades del contexto vital, así como para el ejercicio efectivo de los derechos y deberes ciudadano (Rychen y Salganik, 2001), que hoy denominamos “competencias básicas". La selección de dichas competencias clave en la mayoría de los contextos europeos, se ha realizado siguiendo los tres criterios de DeSeCo (Rychen y Salganick, 2001):

- Que contribuya a obtener resultados de alto valor personal y social.

- Que sean aplicables a un amplio abanico de contextos y ámbitos relevantes.

- Que sean importantes para todas las personas para superar con éxito las exigencias complejas. Es decir, que sean beneficiosas para la totalidad de la población independientemente del sexo, condición social, cultural y entorno familiar.

Siguiendo estos criterios, podemos incluir las competencias emocionales dentro de las competencias básicas para la vida, ya que como hemos visto, contribuyen a la consecución de resultados de alto valor personal y social, son aplicables en todos los ámbitos socioeducativos y facilitan la superación de obstáculos y la consecución de metas, mediante la facilitación de un adecuado funcionamiento. Además, el desarrollo de estas competencias no sólo es beneficiosa para todos los niños/as y docentes, sino que beneficia especialmente a aquellos alumnos con necesidades específicas de atención educativa (Obiakor, 2001; Poeduvicky, Truene y Sperlazza, 2006) y favorece el ajuste intercultural (Yoo, Matsumoto y LeRoux, 2006).

El actual sistema educativo pretende reforzar la formación integral del alumnado y desarrollar en él la adquisición de las capacidades necesarias para intervenir con autonomía, responsabilidad y actitud crítica en una sociedad en permanente cambio, donde el desarrollo personal y su propio bienestar, además de la adquisición de las habilidades culturales básicas relativas a la expresión y comprensión oral, a la lectura, a la escritura y al cálculo, así como el desarrollo de habilidades sociales, hábitos de trabajo y estudio, el sentido artístico, la creatividad y la afectividad son, entre otros, elementos imprescindibles y necesarios para desenvolverse adecuadamente en la sociedad del siglo XXI. 
Para conseguir dichos objetivos educativos, tanto generales como en términos de competencias, la administración presta una atención prioritaria a la formación inicial y permanente del profesorado, cuya reforma debe llevarse a cabo en los próximos años en el contexto del nuevo EEES, con el fin de dar respuesta a las necesidades y a las nuevas demandas que recibe el sistema educativo.

En el Libro Blanco para el Título de Grado de Magisterio (ANECA, 2005), tras un extenso estudio en el que participaron docentes en activo, aparece una selección de competencias que todo titulado en el grado de magisterio, es decir, futuro maestro, debería alcanzar. Según dicho estudio, focalizado en la demanda profesional, el docente de hoy en día deberá mostrar: un buen desarrollo intelectual, moral, emocional y social y ser capaz de promoverlo en la diversidad de su alumnado, además de saber trabajar con toda la comunicad educativa, investigar en su realidad y gestionar adecuadamente los centros.

En la misma línea aparecen descritas las funciones docentes en la legislación educativa vigente para la escolaridad obligatoria (LOE, 2006), mostrando un perfil docente multidimensional, en el que el profesor emerge como un agente educativo dinámico que, en colaboración con el resto de profesionales y familias, desarrolla tareas no sólo de organización y desarrollo de la docencia, sino también de mediación, innovación, gestión, investigación, evaluación y orientación.

El maestro se perfila como el eje central de la comunidad educativa y por tanto como coordinador de toda la red de relaciones interpersonales y procesos educativos que se dan en éste. No es de extrañar por tanto, que las competencias sociales y emocionales sean consideradas a nivel mundial como un aspecto básico en la preparación para la 'sociedad del conocimiento', donde la capacidad de colaboración, comunicación, creatividad y vivir en comunidad son altamente valoradas (Hawkey, 2006).

Esta multiplicidad de roles demandada, lleva a replantearse, necesariamente, la formación inicial del profesorado, tradicionalmente centrada en la enseñanza de conocimientos y procesos específicos, pero no de competencias básicas personales e interpersonales que doten al futuro docente de la autonomía necesaria para afrontar su propio aprendizaje permanente, la resolución de los problemas habituales de su profesión y la consecución de las metas educativas impuestas. Es en este punto donde queremos celebrar la 
inclusión de las competencias básicas en la formación universitaria, pero lamentamos la exclusión explícita de las competencias emocionales como competencias básicas por ser precisamente básicas para el desarrollo exitoso de cualquier actividad relacionada con el ser humano.

\section{Conclusión}

Los resultados de la investigación en torno a las emociones y la IE permiten afirmar que las competencias emocionales son competencias básicas que nos facilitan un adecuado ajuste personal, social, académico y laboral. El objetivo principal de este artículo ha sido llamar la atención sobre la necesidad urgente de incluir explícitamente la formación en competencias emocionales dentro de los objetivos educativos mínimos de la enseñanza obligatoria.

Sin embargo, aunque el consenso sobre la importancia de las competencias emocionales es alto, la implementación de esta formación en la escuela se encuentra con obstáculos, ya que su éxito depende de la implicación y colaboración de toda la comunidad educativa (Elias et al., 1997; Zins, Weissberg, Wang, \& Walberg, 2004). Por ejemplo, de acuerdo con CASEL, las iniciativas para integrar estas competencias en la escuela, requieren de una adecuada formación (que deberá ser eminentemente práctica) de los profesores y gestores además de la consecuente formación de los estudiantes. Esto pone en evidencia no sólo la necesidad de una adecuada formación inicial y permanente del profesorado para favorecer su propia efectividad docente, sino también la necesidad de una estrecha colaboración entre los centros de formación inicial, los centros de formación permanente del profesorado, los centros educativos en particular y la administración.

Sin duda, la formación inicial constituye uno de los elementos básicos en el desarrollo profesional del maestro y constituye un instrumento importante para el logro de la calidad de la enseñanza. Consideramos, además, que los recursos adquiridos en la formación inicial son los que sirven de base para la formación permanente del profesorado, una necesidad constante de todo profesional.

Precisamente ahora, cuando los centros de formación universitaria están ante la formulación de los nuevos planes de estudio que prepararán a los profesionales europeos para 
la sociedad del siglo XXI, es cuando tenemos que decidir cómo formar a los docentes para que puedan dar una respuesta de calidad a la multiplicidad de funciones y demandas educativas descritas. Para ello, deberemos incluir en dichos planes de estudio conducentes al Titulo de Grado en Magisterio las competencias emocionales de forma explícita, no sólo como competencias transversales para el ajuste socio-personal del docente sino también como herramientas docentes específicas para generar entornos adecuados de aprendizaje y de colaboración así como para favorecer el desarrollo emocional de los alumnos. Para esto último, el docente deberá ser capaz de programar actividades educativas y utilizar metodologías dirigidas a este fin.

Por tanto la formación inicial del docente deberá incluir las competencias emocionales si queremos ser coherentes con lo que la investigación nos ha enseñado, con lo que las leyes educativas nos exigen y con el modelo de sociedad europea que perseguimos.

\section{Referencias}

A.A.V.V. (1990). Declaración Mundial sobre educación para todos. "Satisfacción de las necesidades básicas de aprendizaje”. Jomtien, Tailandia.[en línea, 27/11/07]. Disponible en: http://www.oei.es/efa2000jomtien.htm.

A.A.V.V. (2005). Libro Blanco para el Título de Grado en Magisterio. Madrid: ANECA.

Bar-On, R., y Parker, J.D.A. (2000). Handbook of Emotional Intelligence. San Francisco: Jossey.

Birch, S.H. y Ladd, G.W. (1996). Interpersonal relationships in the school environment and children's early school adjustment: The role of teachers and peers. En J. Juvenon y K. Wentzel (Eds.), Social motivation: Understanding children's school adjustment (pp.199-225). New York: Cambridge University Press.

Bless, H. (2000). The interplay of affect and cognition: the mediating role of general knowledge structures. En J.P. Forgas (Ed.), Feeling and thinking. The role of affect in social cognition. Cambridge, Cambridge: University Press.

Bonhert, A.M., Cmic, K.A. y Lim, K.G. (2003). Emotional Competence and Aggressive Behavior in School-Age Children. Journal of Abnormal Child Psychology, 31(1), 79-91.

Brackett, M.A. y Caruso, D.R. (2007). Emotionally literacy for educators. Cary, NC: SELmedia. 
Brackett, M.A., Mayer, J.D. y Warner, R.M. (2004). Emotional intelligence and the prediction of behavior. Personality and Individual Differences, 36, 1387-1402.

Brackett, M., Palomera, R. y Mojsa, J. (en preparación). Emotion regulation ability, burnout and job satisfaction among secondary school teachers.

Brotheridge, C.M. y Grandey, A.A. (2002). Emotional intelligence and burnout: Comparing two perspectives of 'people work'. Journal of Vocational Behavior, 60, 17-39.

Byron, C.M. (2001). The effects of emotional knowledge education in the training of novice teachers. Dissertation Abstract: 2001-95021-143.

Ciarrochi, J., Forgas, J. P., y Mayer, J. D. (Eds.) (2001). Emotional Intelligence in every day life. New York: Psychology Press.

Ciarrochi, J., Forgas, J. P., y Mayer, J. D. (Eds.) (2006). Emotional Intelligence in every day life. Philadelphia: Psychology Press/Taylor and Francis.

Damasio, A.R. (1994). Descarte's error: emotion, reason and the human brain. New York: Putnam.

Diener, E., Sandvik, E., y Pavot, W. (1991). Happiness is the frequency, not the intensity, of positive versus negative affect. En F. Strack, M. Argyle, \& N. Schwarz (Eds.), Subjective well-being: An interdisciplinary perspective (pp.119-139). Oxford, England: Pergamon Press.

Durlak, J. A., y Weissberg, R. P. (2005, August). A major meta-analysis of positive youth development programs. Invited presentation at the Annual Meeting of the American Psychological Association. Washington, DC.

Elias, M., Zins, J., Weissberg, R., Frey, K., Greenberg, T., Haynes, N., Kessler, R., SchwabStone, M., y Shriver, T. (1997). Promoting social and emotional learning: Guidelines for educators. Alexandria, VA: Association for Supervision and Curriculum Development.

Emmer, E.T. (1994). Toward an understanding of the primary of classroom management and discipline. Teaching Education, 6, 65-69.

Eurydice (2002). Las competencias clave: un concepto en expansión dentro de la educación general obligatoria. Madrid: MEC. [en línea 27/11/07]. Disponible en: http://www.eurydice.org.html.

Extremera, N. y Fernández-Berrocal, P. (2005a). Inteligencia emocional y diferencias individuales en el meta-conocimiento de los estados emocionales: una revisión de los estudios con el Trait Meta-Mood Scale. Ansiedad y Estrés, 11, 101-122. 
Extremera, N. y Fernández-Berrocal, P. (2005b). Perceived emotional intelligence and life satisfaction: Predictive and Incremental validity using the Trait Meta-mood Scale. Personality and Individual Differences, 39, 937-948.

Eysenck, M.W. y Calvo, M.G. (1992). Anxiety and performance: the processing efficiency theory. Cognitive Emotion, 6, 409-434.

Fernández-Berrocal, P., Alcaide, R., Extremera, N. y Pizarro, D. (2006). The role of emotional intelligence in anxiety and depression among adolescents. Individual Differences Research, 4(1), 16-27.

Fernández-Berrocal, P., y Extremera, N. (2006). Emotional intelligence: A theoretical and empirical overview of its first 15 years of history. Psicothema, 18, 7-12.

Fernández-Berrocal, P., Extremera, N. y Palomera, R. (2008). Emotional intelligence as a crucial ability on educational context. In A. Valle y J.C. Núñez (Eds.). Handbook of Instructional Resources and Their Applications in the Classroom. NJ: Nova Publishers.

Fernández-Berrocal, P. y Ramos, N. (2002). Corazones inteligentes. Barcelona: Kairós.

Forgas, J.P. (2000). Feeling and thinking, the role of affect in social cognition. Cambridge, Cambridge: University Press.

Frederickson, B.L. (2001). The role of positive emotions in positive psychology: the broadening-and-build theory of positive emotions. American Psychology, 56, 218-226.

Gil-Olarte, P., Palomera, R. y Brackett, M.A. (2006). Relating emotional intelligence to social competence, and academia achievement among high school students. Psicothema, 18(supl.), 118-123.

Gohm, C.L. y Clore, G.L. (2002). Tour latent traits of emotional experience and their involvement in well-being, coping, and attributional style. Cognition and Emotion, 16, 495-518.

Greenberg, M. T, Weissberg, R. P., O'Brien, M. U., Zins, J., Fredericks, L., Resnik H. y Elias, M. J. (2003). Enhancing school-based prevention and youth development through coordinated social, emotional, and academic learning, American Psychologist, 58, 466-474.

Hawkey, K. (2006). Emotional intelligence and mentoring in pre-service teacher education: a literature review. Mentoring \& Tutoring, 14(2), 137-147.

Kuperminc, G.P., Leadbeater, B.J. y Blatt, S.J (2001). School social climate and individual differences in vulnerability to psychopathology among middle school students. Journal of School Psychology, 39 (2), 141-159. 
Kuperminc, G.P., Leadbeater, B.J., Emmons, C.L. y Blatt, S.J (1997). Perceived school climate and difficulties in the social adjustment of middle school students. Applied Developmental Science, 1, 76-88.

Lopes, P.N., Brackett, M.A., Netzlek, J.B., Schütz, Sellin, I. y Salovey, P. (2004). Emotional intelligence and social interaction. Personality and Social Psychology Bulletin, 30(8), 1018-1034.

Lopes, P.N., Salovey, P., Côté, S. y Beers, M. (2005). Emotion regulation ability and the quality of social interaction. Emotion, 5, 113-118.

Lopes, P. N., Salovey, P., y Straus, R. (2003). Emotional intelligence, personality and the perceived quality of social relationships. Personality and Individual Differences, 35, 641-658.

Lupton, D. (1998). The emotional self. London: Sage.

Lyubomirsky, S., Diener, E., y King, L. (2005). The benefits of frequent positive affect: Does happiness lead to success? Psychological Bulletin, 131(6), 803-855.

Mayer, J. D., Caruso, D. y Salovey, P. (1999). Emotional intelligence meets traditional standards for an intelligence. Intelligence, 27, 267-298.

Mayer, J. D. y Salovey, P. (1997). What is emotional intelligence? En P. Salovey y D. Sluyter (Eds). Emotional Development and Emotional Intelligence: Implications for Educators (pp. 3-31). New York: Basic Books.

Mayer, J. D., Salovey, P., y Caruso, D. (2000). Models of emotional intelligence. En R.J. Sternberg (Ed.), Handbook of Intelligence (2nd ed) (pp. 396-420). New York: Cambridge.

Ministerio de Educación y Ciencia (2006). Ley Orgánica 2/2006 de Educación. BOE, 106, de 4 de mayo de 2006.

Mendes, E.J. (2003). The relationship between emotional intelligence and occupational burnout in secondary school teachers. Dissertation Abstracts: 2003-95008-284.

Obiakor, F.E. (2001). Developing emotional intelligence in learners with behavioural problems: Refocusing special education. Behavior Disorders, 26(4), 321-331.

OCDE (2002). Conocimientos y aptitudes para la vida. Primeros resultados del Programa Internacional de Evaluación de Estudiantes (PISA) 2000 de la OCDE. México: Santillana.

Palmer, B., Donaldson, C., y Stough, C. (2002). Emotional intelligence and life satisfaction. Personality and Individual Differences, 33, 1091-1100.

Palomera, R. y Brackett, M.A. (2006). Frequency of positive affect as a possible mediator 
between perceived emotional intelligence and life satisfaction. Ansiedad y Estrés, 12(23), 231-239.

Parker, J.D., Hogan, M.J., Eastabrook, J.M., Oke, A. y Wood, L.M. (2006). Emotional intelligence and student retention: Predicting the successful transition from high school to university. Personality and Individual Differences, 41(7), 1329-1336.

Petrides, K.V., Sangareau, Y., Furnham, A. y Frederickson, N. (2006). Trait emotional intelligence and children's peer relations at school. Social Development, 15(3), 537-547.

Poedubicky, V.A., Truene, L. y Sperlazza, J. (2006). Promoting Social and Emotional Intelligence for Students with Special Needs. En J. Pellitieri, R. Stern, C. Shelton y B. Muller-Ackerman (Eds.), Emotionally intelligent school counselling (pp.125-139). Mahwah, NJ: Lawrence Erlbaum Associates Publishers.

Qualter, P., Whiteley, H.E., Hutchinson, J.M. y Pope, D.J. (2007). Supporting the development of emotional intelligence competencies to ease the transition from primary to high school. Educational Psychology in Practice, 23(1), 79-95.

Roeser, R.W. y Eccles, J.S. (1998). Adolescent's perceptions of middle school. Relation to longitudinal changes in academic and psychological adjustment. Journal of Research on Adolescence, 8, 123-158.

Rychen, D.S. y Salganick, L.H. (2001). Definir y seleccionar las competencias fundamentales para la vida. México: Fondo de Cultura Económica.

Salovey, P. y Mayer, J. D. (1990). Emotional intelligence. Imagination, Cognition, and Personality, 9, 185-211.

Salovey, P., Stroud, L.R., Woolery, A., y Epel, E.S. (2002). Perceived emotional intelligence, stress reactivity, and symptom reports: Further explorations using the Trait Meta-Mood Scale. Psychology and Health, 17, 611-627.

Seligman, M.E.P. (2005). La auténtica felicidad. Barcelona: Ediciones B.

Schutte, N. S., Malouff, J., Bobik, C., Coston, T., Greeson, C., Jedlicka, C., y Wendorf, G. (2001). Emotional intelligence and interpersonal relations. Journal of Social Psychology, 2001, 141, 523-536.

Sutton, R.E. y Harper, E.M. (in press). Teachers' Emotion Regulation. In: L.J., Saha, A.G. Dworkin (eds.) The New International Handbook of Teachers and Teaching. Springer.

Sutton, R.E. y Wheatley, K.F. (2003). Teacher's Emotions and Teaching: A review of the literature and directions for future research. Educational Psychology Review, 15(4), 327-358. 
Trinidad, D.R. y Johnson, C.A. (2002). The association between emotional intelligence and early adolescent tobacco and alcohol use. Personality \& Individual Differences, 32 95105.

Vanderberghe, J. y Huberman, A.M. (Eds.)(1999). Understanding and preventing teacher burnout: A sourcebook of international research and practice. Cambridge, UK: Cambridge University Press.

Weare, K. y Gray, G. (2003). What works in developing children's emotional and social competence and wellbeing? Department for Education and Skills research report (no. 456). London: DfES.

Westling, M. (2002). A two level analysis of classroom climate in relation to social context, group composition and organization of special support. Learning Environments Research, 5, 253-274.

Williams, F.M, Fernández-Berrocal, P., Extremera, N., Ramos, N. y Joiner, T. E. (2004). Mood regulation skill and the symptoms of endogenous and hopelessness depression. Journal of Psychopathology and Behavioral Assessment, 26, 233-240.

Yoo, S.H., Matsumoto, D. y LeRoux, J.A. (2006). The influence of emotion recognition and emotion regulation on intercultural adjustment. International Journal of Intercultural Relations, 30(3), 345-363.

Yoon, J.S. (2002). Teacher characteristics as predictors of teacher-student relationships: stress, negative affect, and self-efficacy. Social Behavior and Personality: An International Journal, 30(5), 485-493.

Zins, J. E., Weissberg, R. P., Wang, M. C., y Walberg, H. J. (Eds.). (2004). Building academic success on social and emotional learning. New York: Teachers College Press 